\title{
Stability of the Diffusion Equation with a Source
}

\author{
Soon-Mo Jung $\mathbb{D}^{1}$ and Seungwook Min $^{2}$ \\ ${ }^{1}$ Mathematics Section, College of Science and Technology, Hongik University, 30016 Sejong, Republic of Korea \\ ${ }^{2}$ Division of Computer Science, Sangmyung University, 03016 Seoul, Republic of Korea
}

Correspondence should be addressed to Soon-Mo Jung; smjung@hongik.ac.kr

Received 13 May 2018; Accepted 11 July 2018; Published 1 August 2018

Academic Editor: Shanhe Wu

Copyright (C) 2018 Soon-Mo Jung and Seungwook Min. This is an open access article distributed under the Creative Commons Attribution License, which permits unrestricted use, distribution, and reproduction in any medium, provided the original work is properly cited.

We will prove the generalized Hyers-Ulam stability of the (inhomogeneous) diffusion equation with a source, $u_{t}(x, t)-k \Delta u(x, t)=$ $f(x, t)$, for a class of scalar functions with continuous second partial derivatives.

\section{Introduction}

The stability problem for functional equations or differential equations started with the well-known question of Ulam [1]: Under what conditions does there exist an additive function near an approximately additive function? In 1941, Hyers [2] gave an affirmative answer to the question of Ulam for the Banach space cases. Indeed, Hyers' theorem states that the following statement is true for all $\varepsilon \geq 0$ : If a function $f$ satisfies the inequality $\|f(x+y)-f(x)-f(y)\| \leq \varepsilon$ for all $x$, then there exists an exact additive function $F$ such that $\|f(x)-F(x)\| \leq \varepsilon$ for all $x$. In that case, the Cauchy additive functional equation, $f(x+y)=f(x)+f(y)$, is said to have (satisfy) the Hyers-Ulam stability.

Assume that $V$ is a normed space and $I$ is an open interval of $\mathbb{R}$. The $n$th order linear differential equation

$$
\begin{aligned}
& a_{n}(x) y^{(n)}(x)+a_{n-1}(x) y^{(n-1)}(x)+\cdots+a_{1}(x) y^{\prime}(x) \\
& +a_{0}(x) y(x)+h(x)=0
\end{aligned}
$$

is said to have (satisfy) the Hyers-Ulam stability provided the following condition is satisfied for all $\varepsilon \geq 0$ : If a function $u$ : $I \longrightarrow V$ satisfies the differential inequality

$$
\begin{aligned}
& \| a_{n}(x) u^{(n)}(x)+a_{n-1}(x) u^{(n-1)}(x)+\cdots+a_{1}(x) u^{\prime}(x) \\
& \quad+a_{0}(x) u(x)+h(x) \| \leq \varepsilon
\end{aligned}
$$

for all $x \in I$, then there exists a solution $u_{0}: I \longrightarrow V$ to the differential equation (1) and a continuous function $K$ such that $\left\|u(x)-u_{0}(x)\right\| \leq K(\varepsilon)$ for any $x \in I$ and $\lim _{\varepsilon \rightarrow 0} K(\varepsilon)=0$.

When the above statement is true even if we replace $\varepsilon$ and $K(\varepsilon)$ by $\varphi(x)$ and $\Phi(x)$, where $\varphi, \Phi: I \longrightarrow[0, \infty)$ are functions not depending on $u$ and $u_{0}$ explicitly, the corresponding differential equation (1) is said to have (satisfy) the generalized Hyers-Ulam stability. (This type of stability is sometimes called the Hyers-Ulam-Rassias stability.)

These terminologies will also be applied for other differential equations and partial differential equations. For more detailed definitions of these terminologies, refer to [1-9].

To the best of our knowledge, Obloza was the first author who investigated the Hyers-Ulam stability of differential equations (see [10,11]): Assume that $g, r:(a, b) \longrightarrow \mathbb{R}$ are continuous functions with $\int_{a}^{b}|g(x)| d x<\infty$. Suppose $\varepsilon$ is an arbitrary positive real number. Obloza proved that there exists a constant $\delta>0$ such that $\left|y(x)-y_{0}(x)\right| \leq \delta$ for all $x \in(a, b)$ whenever a differentiable function $y:(a, b) \longrightarrow \mathbb{R}$ satisfies $\left|y^{\prime}(x)+g(x) y(x)-r(x)\right| \leq \varepsilon$ for all $x \in(a, b)$ and a function $y_{0}:(a, b) \longrightarrow \mathbb{R}$ satisfies $y_{0}^{\prime}(x)+g(x) y_{0}(x)=r(x)$ for all $x \in(a, b)$ and $y(\tau)=y_{0}(\tau)$ for some $\tau \in(a, b)$. Since then, a number of mathematicians have dealt with this subject (see $[3,12])$.

Prástaro and Rassias seem to be the first authors who investigated the Hyers-Ulam stability of partial differential equations (see [13]). Thereafter, Jung and Lee [14] proved 
the Hyers-Ulam stability of the first-order linear partial differential equation of the form

$$
a u_{x}(x, y)+b u_{y}(x, y)+c u(x, y)+d=0,
$$

where $a, b \in \mathbb{R}$ and $c, d \in \mathbb{C}$ are constants with $\mathfrak{R}(c) \neq 0$. As a further step, Hegyi and Jung proved the generalized HyersUlam stability of the diffusion equation on the restricted domain or with an initial condition (see $[15,16])$.

In this paper, applying ideas from $[15,17]$, we investigate the generalized Hyers-Ulam stability of the (inhomogeneous) diffusion equation with a source

$$
u_{t}(x, t)-k \Delta u(x, t)=f(x, t)
$$

for $x \in \mathbb{R}^{n} \backslash\{(0, \ldots, 0)\}$ and $t>0$, where $k$ is a positive constant, $\triangle=\partial^{2} / \partial x_{1}^{2}+\cdots+\partial^{2} / \partial x_{n}^{2}$, and $n$ is a positive integer.

The main advantages of this present paper over the previous works $[15,16]$ are that this paper deals with the inhomogeneous diffusion equation with a source and it describes the behavior of approximate solutions of diffusion equation in the vicinity of origin (roughly speaking, an approximate solution is a solution to a perturbed equation), while the previous works deal with domains not including the vicinity of origin or the homogeneous diffusion equation (without source term).

\section{Preliminaries}

If $u(x, t)$ is a solution to the diffusion equation (4) with $n=1$ and $a$ is a positive constant, then the dilated function $w(x, t):=u(\sqrt{a} x, a t)$ satisfies the equality, $w_{t}(x, t)-$ $k w_{x x}(x, t)=a f(\sqrt{a} x, a t)$, for all $x>0$ and $t>0$. When the source term $f(x, t)$ satisfies the additional condition

$$
a f(\sqrt{a} x, a t)=f(x, t),
$$

$w(x, t)$ is also a solution to (4) with $n=1$. This property is called the invariance under dilation. Hence, it is worth searching for approximate solutions to (4), which are scalar functions of the form

$$
u(x, t)=\frac{1}{(4 k t)^{\alpha}} v\left(\frac{|x|}{\sqrt{4 k t}}\right),
$$

where $\alpha$ is a real parameter which will be determined later and $v$ is a twice continuously differentiable function. That is, $u(x, t)$ depends on $x$ and $t$ primarily through the term $|x| / \sqrt{4 k t}$. We note that the intention of inclusion of the factor $1 / \sqrt{4 k}$ in the above formula is to simplify our formulations later.

Throughout this paper, let $n$ be a fixed positive integer if there is no specification. Each point $x$ in $\mathbb{R}^{n}$ is expressed as $x=\left(x_{1}, \ldots, x_{i}, \ldots, x_{n}\right)$, where $x_{i}$ denotes the $i$ th coordinate of $x$. Moreover, $|x|$ denotes the Euclidean distance of $x$ from the origin; i.e.,

$$
|x|=\sqrt{x_{1}^{2}+\cdots+x_{i}^{2}+\cdots+x_{n}^{2}} .
$$

Based on this argument, we define

$$
U_{\alpha}^{n}:=\left\{u: \mathbb{R}^{n} \backslash\{(0, \ldots, 0)\} \times(0, \infty) \longrightarrow \mathbb{R} \mid\right.
$$

there exists a twice continuously differentiable function $v$ :

$$
\begin{aligned}
& (0, \infty) \longrightarrow \mathbb{R} \text { with } u(x, t)=\frac{1}{(4 k t)^{\alpha}} v\left(\frac{|x|}{\sqrt{4 k t}}\right) \text { for all } x \\
& \left.\in \mathbb{R}^{n} \backslash\{(0, \ldots, 0)\}, t>0\right\},
\end{aligned}
$$

where $n$ is a positive integer and $\alpha$ is a parameter.

The proof of the following lemma runs in the usual and routine way. Hence, we omit the proof.

Lemma 1. If a function $u$ belongs to $U_{\alpha}^{n}$ and a twice continuously differentiable function $v:(0, \infty) \rightarrow \mathbb{R}$ is correspondingly given by

$$
u(x, t)=\frac{1}{(4 k t)^{\alpha}} v\left(\frac{|x|}{\sqrt{4 k t}}\right),
$$

then

$$
\begin{gathered}
u_{t}(x, t)-k \Delta u(x, t)=-\frac{k}{(4 k t)^{\alpha+1}}\left(v^{\prime \prime}(r)\right. \\
\left.+\left(2 r+\frac{n-1}{r}\right) v^{\prime}(r)+4 \alpha v(r)\right)
\end{gathered}
$$

for all $x \in \mathbb{R}^{n} \backslash\{(0, \ldots, 0)\}, t>0$, and for all $r>0$ obeying the relation $r=|x| / \sqrt{4 k t}$.

Let us define the second-order differential operator $\mathscr{L}_{\alpha}^{2}$ : $\mathrm{C}^{2}(0, \infty) \rightarrow \mathrm{C}(0, \infty)$ by

$$
\mathscr{L}_{\alpha}^{2} v(r):=v^{\prime \prime}(r)+\left(2 r+\frac{n-1}{r}\right) v^{\prime}(r)+4 \alpha v(r),
$$

where $C(0, \infty)$ and $C^{2}(0, \infty)$ denote the set of all continuous real-valued functions and the set of all twice continuously differentiable real-valued functions defined on $(0, \infty)$, respectively.

We now try to decompose the differential operator $\mathscr{L}_{\alpha}^{2}$ into differential operators $\mathscr{L}_{A(r)}$ and $\mathscr{L}_{B(r)}$ of first order such that

$$
\mathscr{L}_{\alpha}^{2} v(r)=\left(\mathscr{L}_{A(r)} \circ \mathscr{L}_{B(r)}\right) v(r)
$$

for all $v \in C^{2}(0, \infty)$, where we define

$$
\begin{aligned}
& \mathscr{L}_{A(r)} v(r):=v^{\prime}(r)+A(r) v(r), \\
& \mathscr{L}_{B(r)} v(r):=v^{\prime}(r)+B(r) v(r) .
\end{aligned}
$$

Then we have

$$
\begin{aligned}
\left(\mathscr{L}_{A(r)} \circ \mathscr{L}_{B(r)}\right) v(r)= & v^{\prime \prime}(r)+(A(r)+B(r)) v^{\prime}(r) \\
& +\left(A(r) B(r)+B^{\prime}(r)\right) v(r) .
\end{aligned}
$$


Comparing both (12) and (14), we obtain

$$
\begin{aligned}
A(r)+B(r) & =2 r+\frac{n-1}{r}, \\
A(r) B(r)+B^{\prime}(r) & =4 \alpha .
\end{aligned}
$$

From the last system of equations, we get a Riccati equation

$$
B^{\prime}(r)+\left(2 r+\frac{n-1}{r}\right) B(r)-B(r)^{2}=4 \alpha,
$$

one of whose solutions has the form $(n-1) \beta / r$, where $\beta \neq 0$ is a real constant: If we put $B_{p}(r)=(n-1) \beta / r$ in the Riccati equation (16), then we have

$$
\begin{aligned}
B_{p}^{\prime}(r)+\left(2 r+\frac{n-1}{r}\right) B_{p}(r)-B_{p}(r)^{2} \\
\quad=2(n-1) \beta+\frac{(n-1) \beta(n-2-(n-1) \beta)}{r^{2}} .
\end{aligned}
$$

Comparing (16) with (17) and considering that $4 \alpha$ is a constant, we conclude that

$$
\begin{array}{r}
\alpha=\frac{n-2}{2}, \\
\beta=\frac{n-2}{n-1}, \\
B_{p}(r)=\frac{n-2}{r}
\end{array}
$$

for all integers $n \geq 2$. (Even if $\beta$ is not defined for $n=1$, we can also verify the truth of the formulas for $\alpha$ and $B_{p}(r)$ for $n=1$ by a direct calculation.)

Using this particular solution $B_{p}(r)$ and in view of $[18, \S$ 1.2.1], the general solution of the Riccati equation (16) with $4 \alpha=2 n-4$ is given by

$$
B(r)=\frac{n-2}{r}+\frac{r^{n-3} e^{-r^{2}}}{C_{n}-\int_{r_{0}}^{r} \omega^{n-3} e^{-\omega^{2}} d \omega},
$$

where $r_{0}$ is a nonnegative fixed real number, $C_{n}$ is a constant, and we set $C_{n}=\infty$ for the particular solution $B_{p}(r)=(n-$ 2) $/ r$.

Lemma 2. Let $n$ be a positive integer. Then

$$
\mathscr{L}_{(n-2) / 2}^{2} v(r)=\left(\mathscr{L}_{2 r+(n-1) / r-B(r)} \circ \mathscr{L}_{B(r)}\right) v(r)
$$

for all $v \in \mathrm{C}^{2}(0, \infty)$, where $B(r)$ is defined in (19).

\section{Main Results}

Before starting with our main theorem, we modify the theorem ([19, Theorem 1]) into a more suitable form for practical applications and we will apply this modified version to the proof of our main theorem (cf. [20, Theorem 2.2]). Indeed, the hypotheses of the original theorem [19, Theorem 1] were formulated with $a$ instead of $a_{0}$ which impose a constraint on its usability. The proof of Theorem 3 exactly follows the lines of the proof of [19, Theorem 1]. Hence, we omit the proof.
Theorem 3 ([19, Theorem 1, Remark 3]). Assume that $X$ is a real Banach space and $I=(a, b)$ is an open interval for arbitrary constants $a, b \in \mathbb{R} \cup\{ \pm \infty\}$ with $a<b$. Let $p: I \longrightarrow \mathbb{R}$ and $q: I \longrightarrow X$ be continuous functions such that there exists a constant $a_{0} \in[a, b)$ with the following properties:

(i) $\int_{a_{0}}^{t} p(s) d s$ exists for each $t \in I$.

(ii) $\int_{a_{0}}^{t} q(y) \exp \left\{\int_{a_{0}}^{y} p(s) d s\right\} d y$ exists for any $t \in I$.

Moreover, assume that $\varphi: I \longrightarrow[0, \infty)$ is a function such that

$$
\text { (iii) } \int_{a_{0}}^{b} \varphi(y) \exp \left\{\int_{a_{0}}^{y} p(s) d s\right\} d y \text { exists. }
$$

If a continuously differentiable function $v: I \longrightarrow X$ satisfies the differential inequality

$$
\left\|v^{\prime}(t)+p(t) v(t)+q(t)\right\| \leq \varphi(t)
$$

for all $t \in I$, then there exists a unique continuously differentiable function $v_{0}: I \longrightarrow X$ such that $v_{0}^{\prime}(t)+p(t) v_{0}(t)+q(t)=0$ for all $t \in I$ and

$$
\begin{aligned}
& \left\|v(t)-v_{0}(t)\right\| \\
& \leq \exp \left\{-\int_{a_{0}}^{t} p(s) d s\right\} \int_{t}^{b} \varphi(y) \exp \left\{\int_{a_{0}}^{y} p(s) d s\right\} d y
\end{aligned}
$$

for all $t \in I$.

The following theorem is the main result of this paper which deals with the generalized Hyers-Ulam stability of the diffusion equation (4) when $n$ is an integer larger than 2 .

Theorem 4. Let $n \geq 3$ be an integer and assume that $\varphi, \psi$ : $(0, \infty) \longrightarrow[0, \infty)$ are functions satisfying the conditions

$$
\int_{0}^{\infty} z e^{z^{2}} \varphi(z) d z<\infty
$$

and

$$
c:=\inf _{t>0} \frac{(4 k t)^{n / 2}}{k} \psi(t)>0 .
$$

Suppose $f: \mathbb{R}^{n} \backslash\{(0, \ldots, 0)\} \times(0, \infty) \longrightarrow \mathbb{R}$ is a function for which there exists a continuous function $g:(0, \infty) \longrightarrow \mathbb{R}$ such that

$$
f(x, t)=\frac{k}{(4 k t)^{n / 2}} g\left(\frac{|x|}{\sqrt{4 k t}}\right)
$$

for all $x \in \mathbb{R}^{n} \backslash\{(0, \ldots, 0)\}$ and $t>0$ and

$$
\int_{0}^{\infty} z e^{z^{2}}|g(z)| d z<\infty
$$

Assume moreover that the constant $C_{n}$ in (19) is chosen such that

$$
m \int_{0}^{\infty} \omega^{n-3} e^{-\omega^{2}} d \omega \leq C_{n}<(m+1) \int_{0}^{\infty} \omega^{n-3} e^{-\omega^{2}} d \omega
$$


TABle 1

\begin{tabular}{llllllllll}
\hline Theorem 3 & $X$ & $t$ & $a$ & $b$ & $a_{0}$ & $v(t)$ & $p(t)$ & $\varphi(t)$ \\
\hline$(33)$ & $\mathbb{R}$ & $r$ & 0 & $\infty$ & $r_{0}$ & $w(r)$ & $2 r+\frac{n-1}{r}-B(r)$ & $g(t)$ & $c \varphi(r)$ \\
\hline
\end{tabular}

for some integer $m \geq 2$. For any function $u \in U_{(n-2) / 2}^{n}$ satisfying the inequality

$$
\left|u_{t}(x, t)-k \Delta u(x, t)-f(x, t)\right| \leq \varphi\left(\frac{|x|}{\sqrt{4 k t}}\right) \psi(t)
$$

for all $x \in \mathbb{R}^{n} \backslash\{(0, \ldots, 0)\}$ and $t>0$, there exists a solution $u_{0}: \mathbb{R}^{n} \backslash\{(0, \ldots, 0)\} \times(0, \infty) \longrightarrow \mathbb{R}$ of the diffusion equation (4) such that $u_{0} \in U_{(n-2) / 2}^{n}$ and

$$
\begin{aligned}
& \left|u(x, t)-u_{0}(x, t)\right| \\
& \quad \leq \frac{c e^{2 /(m-1)}}{|x|^{n-2}} \int_{0}^{\infty} z e^{z^{2}} \varphi(z) d z \int_{0}^{\infty} y^{n-3} e^{-y^{2}} d y
\end{aligned}
$$

for all $x \in \mathbb{R}^{n} \backslash\{(0, \ldots, 0)\}$ and $t>0$.

Proof. Since $u \in U_{(n-2) / 2}^{n}$, it follows from Lemma 1 with $\alpha=$ $(n-2) / 2,(25)$, and (28) that

$$
\begin{aligned}
& \left|u_{t}(x, t)-k \Delta u(x, t)-f(x, t)\right|=\frac{k}{(4 k t)^{n / 2}} \mid v^{\prime \prime}(r) \\
& \quad+\left(2 r+\frac{n-1}{r}\right) v^{\prime}(r)+(2 n-4) v(r)+g(r) \mid \\
& \quad \leq \varphi(r) \psi(t)
\end{aligned}
$$

for all $x \in \mathbb{R}^{n} \backslash\{(0, \ldots, 0)\}$ and $t>0$, where $v:(0, \infty) \longrightarrow \mathbb{R}$ is the twice continuously differentiable function given in the definition of $U_{(n-2) / 2}^{n}$ and we set $r=|x| / \sqrt{4 k t}$. In view of (11), (24), and the last inequality, we have

$$
\begin{aligned}
& \left|\mathscr{L}_{(n-2) / 2}^{2} v(r)+g(r)\right|=\mid v^{\prime \prime}(r)+\left(2 r+\frac{n-1}{r}\right) v^{\prime}(r) \\
& +(2 n-4) v(r)+g(r) \mid \leq c \varphi(r)
\end{aligned}
$$

for all $r>0$. We here note that $\left\{r: x \in \mathbb{R}^{n} \backslash\{(0, \ldots, 0)\}, t>\right.$ $0\}=(0, \infty)$.

On account of Lemma 2, we further have

$$
\left|\left(\mathscr{L}_{2 r+(n-1) / r-B(r)} \circ \mathscr{L}_{B(r)}\right) v(r)+g(r)\right| \leq c \varphi(r)
$$

for all $r>0$, where $B(r)$ is defined in (19). If we define a continuously differentiable function $w:(0, \infty) \longrightarrow \mathbb{R}$ by $w(r):=\mathscr{L}_{B(r)} v(r)=v^{\prime}(r)+B(r) v(r)$, then it follows from the last inequality that

$$
\left|w^{\prime}(r)+\left(2 r+\frac{n-1}{r}-B(r)\right) w(r)+g(r)\right| \leq c \varphi(r)
$$

for all $r>0$, where $B(r)$ is given in (19) with a positive real constant $r_{0}$.

We can now apply Theorem 3 to our inequality (33) by considering the substitutions as we see in Table 1.
Our hypothesis that $n$ is an integer not less than 3 implies that

$$
\int_{0}^{\infty} \omega^{n-3} e^{-\omega^{2}} d \omega<\infty
$$

It then follows from (19) and (27) that

$$
\begin{gathered}
\frac{n-2}{s}+\frac{s^{n-3} e^{-s^{2}}}{(m+2) \int_{0}^{\infty} \omega^{n-3} e^{-\omega^{2}} d \omega}<B(s) \\
<\frac{n-2}{s}+\frac{s^{n-3} e^{-s^{2}}}{(m-1) \int_{0}^{\infty} \omega^{n-3} e^{-\omega^{2}} d \omega} .
\end{gathered}
$$

Hence, we have

$$
\begin{aligned}
\int_{r_{0}}^{r} p(s) d s= & \int_{r_{0}}^{r}\left(2 s+\frac{n-1}{s}-B(s)\right) d s \\
< & r^{2}-r_{0}^{2}+\ln r-\ln r_{0} \\
& -\frac{\int_{r_{0}}^{r} s^{n-3} e^{-s^{2}} d s}{(m+2) \int_{0}^{\infty} \omega^{n-3} e^{-\omega^{2}} d \omega} \\
& <r^{2}-r_{0}^{2}+\ln r-\ln r_{0}+\frac{1}{m+2},
\end{aligned}
$$

which implies that the condition ( $i$ ) of Theorem 3 is satisfied.

Moreover, it follows from the last inequality that

$$
\exp \left\{\int_{r_{0}}^{y} p(s) d s\right\}<\frac{e^{1 /(m+2)}}{r_{0} e^{r_{0}^{2}}} y e^{y^{2}}
$$

and, by (26), we get

$$
\begin{aligned}
& \int_{r_{0}}^{r} \exp \left\{\int_{r_{0}}^{y} p(s) d s\right\}|g(y)| d y \\
& \quad<\frac{e^{1 /(m+2)}}{r_{0} e^{r_{0}^{2}}} \int_{r_{0}}^{r} y e^{y^{2}}|g(y)| d y<\infty
\end{aligned}
$$

for all $r>0$, which means that the condition (ii) of Theorem 3 is satisfied.

Similarly, it also follows from (23) that

$$
\begin{aligned}
& \int_{r_{0}}^{\infty} c \varphi(y) \exp \left\{\int_{r_{0}}^{y} p(s) d s\right\} d y \\
& <\frac{c e^{1 /(m+2)}}{r_{0} e^{r_{0}^{2}}} \int_{r_{0}}^{\infty} y e^{y^{2}} \varphi(y) d y<\infty,
\end{aligned}
$$

by which we conclude that the condition (iii) of Theorem 3 is satisfied. 
TABLE 2

\begin{tabular}{llllllllll}
\hline Theorem 3 & $X$ & $t$ & $a$ & $b$ & $a_{0}$ & $v(t)$ & $p(t)$ & $q(t)$ & $\varphi(t)$ \\
\hline$(45)$ & $\mathbb{R}$ & $r$ & 0 & $\infty$ & $r_{0}$ & $v(r)$ & $B(r)$ & $-w_{0}(r)$ & $c \int_{r}^{\infty} \frac{y e^{y^{2}}}{r e^{r^{2}}} \varphi(y) d y$ \\
\hline
\end{tabular}

According to Theorem 3 (or [19, Theorem 1]) and (33), there exists a unique continuously differentiable function $w_{0}$ : $(0, \infty) \longrightarrow \mathbb{R}$ such that

$$
w_{0}^{\prime}(r)+\left(2 r+\frac{n-1}{r}-B(r)\right) w_{0}(r)+g(r)=0
$$

for all $r>0$ and

$$
\begin{aligned}
& \left|w(r)-w_{0}(r)\right| \leq \exp \left\{-\int_{r_{0}}^{r} p(s) d s\right\} \\
& \cdot \int_{r}^{\infty} c \varphi(y) \exp \left\{\int_{r_{0}}^{y} p(s) d s\right\} d y \\
& \quad=c \int_{r}^{\infty} \frac{y^{n-1} e^{y^{2}}}{r^{n-1} e^{r^{2}}} \varphi(y) \exp \left\{-\int_{r}^{y} B(s) d s\right\} d y
\end{aligned}
$$

for all $r>0$. In particular, by [19, Theorem 1], $w_{0}(r)$ is explicitly given by

$$
\begin{aligned}
w_{0}(r)= & \sigma \frac{r_{0}^{n-1} e^{r_{0}^{2}}}{r^{n-1} e^{r^{2}}} \exp \left\{\int_{r_{0}}^{r} B(s) d s\right\} \\
& -\int_{r_{0}}^{r} \frac{z^{n-1} e^{z^{2}}}{r^{n-1} e^{r^{2}}} \exp \left\{-\int_{r}^{z} B(s) d s\right\} g(z) d z
\end{aligned}
$$

with some $\sigma \in \mathbb{R}$.

By (35), we have

$$
\begin{gathered}
-B(s)<-\frac{n-2}{s} \\
\int_{y}^{r} B(s) d s<\ln \left(\frac{r}{y}\right)^{n-2}
\end{gathered}
$$

for any $r, y>0$ with $r \leq y$. Therefore, since $r \leq y<\infty$, it follows from (41) that

$$
\left|w(r)-w_{0}(r)\right| \leq c \int_{r}^{\infty} \frac{y e^{y^{2}}}{r e^{r^{2}}} \varphi(y) d y
$$

or

$$
\left|v^{\prime}(r)+B(r) v(r)-w_{0}(r)\right| \leq c \int_{r}^{\infty} \frac{y e^{y^{2}}}{r e^{r^{2}}} \varphi(y) d y
$$

for all $r>0$.

We apply Theorem 3 to our inequality (45) by considering the substitutions as we see in Table 2.

First, in view of (19) and (35), we get

$$
-\frac{1}{m-1}<\int_{r_{0}}^{r} \frac{s^{n-3} e^{-s^{2}}}{C_{n}-\int_{r_{0}}^{s} \omega^{n-3} e^{-\omega^{2}} d \omega} d s<\frac{1}{m-1}
$$

and hence

$$
\begin{aligned}
\ln \left(\frac{r}{r_{0}}\right)^{n-2}-\frac{1}{m-1} & <\int_{r_{0}}^{r} B(s) d s \\
& <\ln \left(\frac{r}{r_{0}}\right)^{n-2}+\frac{1}{m-1}
\end{aligned}
$$

for all $r>0$, by which we see that the condition $(i)$ of Theorem 3 is satisfied.

Further, it follows from the last inequality that

$$
\exp \left\{\int_{r_{0}}^{r} B(s) d s\right\}<\left(\frac{r}{r_{0}}\right)^{n-2} e^{1 /(m-1)}
$$

for any $r, r_{0}>0$. By (42) and (48), we easily get

$$
\begin{aligned}
& \left|w_{0}(r)\right| \\
& \leq|\sigma| \frac{r_{0}^{n-1} e^{r_{0}^{2}}}{r^{n-1} e^{r^{2}}} \exp \left\{\int_{r_{0}}^{r} B(s) d s\right\} \\
& \quad+\int_{0}^{\infty} \frac{z^{n-1} e^{z^{2}}}{r^{n-1} e^{r^{2}}} \exp \left\{-\int_{r}^{z} B(s) d s\right\}|g(z)| d z \\
& \leq|\sigma| \frac{r_{0}^{n-1} e^{r_{0}^{2}}}{r^{n-1} e^{r^{2}}}\left(\frac{r}{r_{0}}\right)^{n-2} e^{1 /(m-1)} \\
& \quad+\int_{0}^{\infty} \frac{z^{n-1} e^{z^{2}}}{r^{n-1} e^{r^{2}}}\left(\frac{r}{z}\right)^{n-2} e^{1 /(m-1)}|g(z)| d z \\
& =\frac{e^{1 /(m-1)}}{r e^{r^{2}}}\left(|\sigma| r_{0} e^{r_{0}^{2}}+\int_{0}^{\infty} z e^{z^{2}}|g(z)| d z\right) .
\end{aligned}
$$

It now follows from (26), (48), and (49) that

$$
\begin{aligned}
& \left|\int_{r_{0}}^{r} \exp \left\{\int_{r_{0}}^{y} B(s) d s\right\}\left(-w_{0}(y)\right) d y\right| \\
& \quad \leq \int_{0}^{\infty} \exp \left\{\int_{r_{0}}^{y} B(s) d s\right\}\left|w_{0}(y)\right| d y \\
& \quad \leq \frac{e^{2 /(m-1)}}{r_{0}^{n-2}}\left(|\sigma| r_{0} e^{r_{0}^{2}}+\int_{0}^{\infty} z e^{z^{2}}|g(z)| d z\right) \\
& \quad \cdot \int_{0}^{\infty} y^{n-3} e^{-y^{2}} d y<\infty,
\end{aligned}
$$

which means that the condition (ii) of Theorem 3 is satisfied. 
Analogously, it follows from (23) and (48) that

$$
\begin{aligned}
& \int_{r_{0}}^{\infty} \frac{c}{y e^{y^{2}}}\left(\int_{y}^{\infty} z e^{z^{2}} \varphi(z) d z\right) \exp \left\{\int_{r_{0}}^{y} B(s) d s\right\} d y \\
& \leq \int_{r_{0}}^{\infty} \frac{c}{y e^{y^{2}}}\left(\int_{0}^{\infty} z e^{z^{2}} \varphi(z) d z\right) \frac{y^{n-2}}{r_{0}^{n-2}} e^{1 /(m-1)} d y \\
& \leq \frac{c e^{1 /(m-1)}}{r_{0}^{n-2}} \int_{0}^{\infty} z e^{z^{2}} \varphi(z) d z \int_{0}^{\infty} y^{n-3} e^{-y^{2}} d y<\infty,
\end{aligned}
$$

by which we conclude that the condition (iii) of Theorem 3 is satisfied.

Due to Theorem 3, (45), and (48), there exists a unique continuously differentiable function $v_{0}:(0, \infty) \longrightarrow \mathbb{R}$ such that

$$
v_{0}^{\prime}(r)+B(r) v_{0}(r)-w_{0}(r)=0
$$

for all $r>0$ and

$$
\begin{aligned}
& \left|v(r)-v_{0}(r)\right| \leq \exp \left\{-\int_{r_{0}}^{r} B(s) d s\right\} \\
& \cdot \int_{r}^{\infty} \frac{c}{y e^{y^{2}}}\left(\int_{y}^{\infty} z e^{z^{2}} \varphi(z) d z\right) \\
& \cdot \exp \left\{\int_{r_{0}}^{y} B(s) d s\right\} d y \leq \frac{r_{0}^{n-2} e^{1 /(m-1)}}{r^{n-2}} \\
& . \frac{c e^{1 /(m-1)}}{r_{0}^{n-2}} \int_{0}^{\infty} z e^{z^{2}} \varphi(z) d z \int_{0}^{\infty} y^{n-3} e^{-y^{2}} d y \\
& \leq \frac{c e^{2 /(m-1)}}{r^{n-2}} \int_{0}^{\infty} z e^{z^{2}} \varphi(z) d z \int_{0}^{\infty} y^{n-3} e^{-y^{2}} d y
\end{aligned}
$$

for all $r>0$. In particular, by [19, Theorem 1$], v_{0}(r)$ is explicitly given by

$$
\begin{gathered}
v_{0}(r)=\tau \exp \left\{-\int_{r_{0}}^{r} B(s) d s\right\}+\exp \left\{-\int_{r_{0}}^{r} B(s) d s\right\} \\
\cdot \int_{r_{0}}^{r} \exp \left\{\int_{r_{0}}^{y} B(s) d s\right\} w_{0}(y) d y
\end{gathered}
$$

with some $\tau \in \mathbb{R}$. We remark that $v_{0}(r)$ is indeed a twice continuously differentiable function.

Now, let us define the twice continuously differentiable function $u_{0}: \mathbb{R}^{n} \backslash\{(0, \ldots, 0)\} \times(0, \infty) \longrightarrow \mathbb{R}$ by

$$
u_{0}(x, t):=\frac{1}{(4 k t)^{(n-2) / 2}} v_{0}(r)
$$

where we set $r=|x| / \sqrt{4 k t}$. Then, $u_{0} \in U_{(n-2) / 2}^{n}$ and inequality (29) follows directly from (53). By Lemma 1 with $(n-2) / 2$ and $u_{0}$ and $v_{0}$ in place of $\alpha, u$, and $v$, respectively, we further have

$$
\begin{gathered}
\frac{\partial}{\partial t} u_{0}(x, t)-k \sum_{i=1}^{n} \frac{\partial^{2}}{\partial x_{i}^{2}} u_{0}(x, t)=-\frac{k}{(4 k t)^{n / 2}}\left(v_{0}^{\prime \prime}(r)\right. \\
\left.+\left(2 r+\frac{n-1}{r}\right) v_{0}^{\prime}(r)+2(n-2) v_{0}(r)\right)
\end{gathered}
$$

for all $x \in \mathbb{R}^{n} \backslash\{(0, \ldots, 0)\}, t>0$, and for all $r>0$ obeying the relation $r=|x| / \sqrt{4 k t}$.

Finally, it follows from Lemma 2, (11), (13), (19), (40), and (52) that

$$
\begin{aligned}
v_{0}^{\prime \prime} & (r)+\left(2 r+\frac{n-1}{r}\right) v_{0}^{\prime}(r)+2(n-2) v_{0}(r) \\
& =\mathscr{L}_{(n-2) / 2}^{2} v_{0}(r)=\left(\mathscr{L}_{2 r+(n-1) / r-B(r)} \circ \mathscr{L}_{B(r)}\right) v_{0}(r) \\
& =\mathscr{L}_{2 r+(n-1) / r-B(r)} w_{0}(r)=-g(r)
\end{aligned}
$$

for all $r>0$. Hence, by (25), we have

$$
\frac{\partial}{\partial t} u_{0}(x, t)-k \sum_{i=1}^{n} \frac{\partial^{2}}{\partial x_{i}^{2}} u_{0}(x, t)=f(x, t)
$$

for all $x \in \mathbb{R}^{n} \backslash\{(0, \ldots, 0)\}$ and $t>0$.

If we set $B(r)=B_{p}(r)=(n-2) / r$ in the proof of Theorem 4 , i.e., if we set $C_{n}=\infty$ in (19), then we obtain the following corollary by letting $m \longrightarrow \infty$ in (27) and (29) of Theorem 4.

Corollary 5. Under the same hypotheses and conditions of Theorem 4 except condition (27), there exists a solution $u_{0}$ : $\mathbb{R}^{n} \backslash\{(0, \ldots, 0)\} \times(0, \infty) \longrightarrow \mathbb{R}$ of the diffusion equation (4) such that $u_{0} \in U_{(n-2) / 2}^{n}$ and

$$
\begin{aligned}
& \left|u(x, t)-u_{0}(x, t)\right| \\
& \quad \leq \frac{c}{|x|^{n-2}} \int_{0}^{\infty} z e^{z^{2}} \varphi(z) d z \int_{0}^{\infty} y^{n-3} e^{-y^{2}} d y
\end{aligned}
$$

for all $x \in \mathbb{R}^{n} \backslash\{(0, \ldots, 0)\}$ and $t>0$.

We now introduce a concrete example for Theorem 4 and Corollary 5 in the following corollary.

Corollary 6. Let $n \geq 3$ be an integer and let $c, \mu_{1}, \mu_{2}, \nu_{1}, \nu_{2}$ be positive real constants and let a function $u: \mathbb{R}^{n} \backslash\{(0, \ldots, 0)\} \times$ $(0, \infty) \longrightarrow \mathbb{R}$ belong to $U_{(n-2) / 2}^{n}$. If the function $u$ satisfies the inequality

$$
\begin{aligned}
& \mid u_{t}(x, t)-k \Delta u(x, t) \\
& \quad-\frac{\mu_{2} k}{|x|(4 k \mathrm{t})^{(n-1) / 2}} \exp \left\{-\frac{|x|^{2}}{4 k t}-v_{2} \frac{|x|}{\sqrt{4 k t}}\right\} \mid \\
& \quad \leq \frac{c \mu_{1} k}{|x|(4 k t)^{(n-1) / 2}} \exp \left\{-\frac{|x|^{2}}{4 k t}-v_{1} \frac{|x|}{\sqrt{4 k t}}\right\}
\end{aligned}
$$

for all $x \in \mathbb{R}^{n} \backslash\{(0, \ldots, 0)\}$ and $t>0$, then there exists a solution $u_{0}: \mathbb{R}^{n} \backslash\{(0, \ldots, 0)\} \times(0, \infty) \longrightarrow \mathbb{R}$ of the diffusion equation (4) such that $u_{0} \in U_{(n-2) / 2}^{n}$ and

$$
\left|u(x, t)-u_{0}(x, t)\right| \leq \frac{c \mu_{1}}{v_{1}|x|^{n-2}} \int_{0}^{\infty} y^{n-3} e^{-y^{2}} d y
$$

for all $x \in \mathbb{R}^{n} \backslash\{(0, \ldots, 0)\}$ and $t>0$. 
Proof. First, we set

$$
\begin{aligned}
\varphi(r) & :=\mu_{1} \frac{e^{-v_{1} r}}{r e^{r^{2}}}, \\
\psi(t) & :=\frac{c k}{(4 k t)^{n / 2}}, \\
f(x, t) & :=\frac{\mu_{2} k}{|x|(4 k t)^{(n-1) / 2}} \exp \left\{-\frac{|x|^{2}}{4 k t}-v_{2} \frac{|x|}{\sqrt{4 k t}}\right\}, \\
g(r) & :=\mu_{2} \frac{e^{-v_{2} r}}{r e^{r^{2}}} .
\end{aligned}
$$

Then, all the conditions and hypotheses (23), (24), (25), (26), and (28) are fulfilled.

Due to Corollary 5 , there exists a solution $u_{0}: \mathbb{R}^{n} \backslash$ $\{(0, \ldots, 0)\} \times(0, \infty) \longrightarrow \mathbb{R}$ of the diffusion equation (4) such that $u_{0} \in U_{(n-2) / 2}^{n}$ and

$$
\begin{aligned}
& \left|u(x, t)-u_{0}(x, t)\right| \\
& \quad \leq \frac{c}{|x|^{n-2}} \int_{0}^{\infty} z e^{z^{2}} \varphi(z) d z \int_{0}^{\infty} y^{n-3} e^{-y^{2}} d y \\
& \quad \leq \frac{c \mu_{1}}{v_{1}|x|^{n-2}} \int_{0}^{\infty} y^{n-3} e^{-y^{2}} d y
\end{aligned}
$$

for all $x \in \mathbb{R}^{n} \backslash\{(0, \ldots, 0)\}$ and $t>0$.

\section{Discussions and Conclusions}

The diffusion equation is sometimes called a heat equation or a continuity equation and it plays an important role in a number of fields of science. For example, the diffusion equation describes the conduction of heat, the signal transmission in communication systems, and diffusion models of chemical diffusion phenomena and it is also connected with Brownian motion in probability theory.

This paper was partially motivated by a previous work [21] in which the generalized Hyers-Ulam stability of the one-dimensional wave equation with a source, $u_{t t}(x, t)-$ $c^{2} u_{x x}(x, t)=f(x, t)$ was investigated by using the method of characteristic coordinates. On the other hand, we prove in this paper the generalized Hyers-Ulam stability of the $n$-dimensional diffusion equation with a source, $u_{t}(x, t)-$ $k \Delta u(x, t)=f(x, t)$, by applying a kind of method for decomposition of differential operators.

The main advantages of this present paper over the existing results $[15,16]$ are that this paper deals with the (inhomogeneous) diffusion equation with a source and it can describe the behavior of approximate solutions of (inhomogeneous) diffusion equation in the vicinity of origin, while the previous work [15] deals with the case of domain $D=\{x \in$ $\left.\mathbb{R}^{n}|a<| x \mid<b\right\}$ with $0<a<b \leq \infty$ and $n \geq 2$, as we see that the domain $D$ does not include the vicinity of origin and while the other existing result [16] deals with the generalized Hyers-Ulam stability of the homogeneous diffusion equation with an initial condition (but without source term).

\section{Data Availability}

The data used to support the findings of this study are included within the article.

\section{Conflicts of Interest}

The authors declare that there are no conflicts of interest regarding the publication of this article.

\section{Authors' Contributions}

All authors contributed equally to the writing of this paper. All authors read and approved the final manuscript.

\section{Acknowledgments}

This research was supported by Basic Science Research Program through the National Research Foundation of Korea (NRF) funded by the Ministry of Education (no. 2016R1D1A1B03931061). This work was supported by 2018 Hongik University Research Fund.

\section{References}

[1] S. M. Ulam, Problems in Modern Mathematics, Wiley, New York, NY, USA, 1964.

[2] D. H. Hyers, "On the stability of the linear functional equation," Proceedings of the National Acadamy of Sciences of the United States of America, vol. 27, pp. 222-224, 1941.

[3] C. Alsina and R. Ger, "On some inequalities and stability results related to the exponential function," Journal of Inequalities and Applications, vol. 2, pp. 373-380, 1998.

[4] J. Brzdek, D. Popa, and I. Rasa, "Hyers-Ulam stability with respect to gauges," Journal of Mathematical Analysis and Applications, vol. 453, no. 1, pp. 620-628, 2017.

[5] J. Brzdek, D. Popa, I. Rasa, and B. Xu, Ulam Stability of Operators, vol. 1 of Mathematical Analysis and Its Applications, Academic Press, Elsevier, 2018.

[6] D. H. Hyers, G. Isac, and T. M. Rassias, Stability of Functional Equations in Several Variables, Birkhäuser, Boston, Mass, USA, 1998.

[7] S.-M. Jung, Hyers-Ulam-Rassias Stability of Functional Equations in Nonlinear Analysis, vol. 48 of Springer Optimization and Its Applications, Springer, New York, NY, USA, 2011.

[8] Z. Moszner, "Stability has many names," Aequationes Mathematicae, vol. 90, no. 5, pp. 983-999, 2016.

[9] Th. M. Rassias, "On the stability of the linear mapping in Banach spaces," Proceedings of the American Mathematical Society, vol. 72, no. 2, pp. 297-300, 1978.

[10] M. Obłoza, "Hyers stability of the linear differential equation," Rocznik Naukowo-Dydaktyczny - Wyższa Szkoła Pedagogiczna im. Komisji Edukacji Narodowej. Prace Matematyczne, no. 13, pp. 259-270, 1993.

[11] M. Obłoza, "Connections between Hyers and Lyapunov stability of the ordinary differential equations," Rocznik NaukowoDydaktyczny - Wyższa Szkoła Pedagogiczna im. Komisji Edukacji Narodowej. Prace Matematyczne, no. 14, pp. 141-146, 1997.

[12] S. E. Takahasi, T. Miura, and S. Miyajima, "On the Hyers-Ulam stability of the Banach space-valued differential equation $y^{\prime}=$ $\lambda y$," Bulletin of the Korean Mathematical Society, vol. 39, no. 2, pp. 309-315, 2002. 
[13] A. Prástaro and T. M. Rassias, "Ulam stability in geometry of PDE's," Nonlinear Functional Analysis and Applications. An International Mathematical Journal for Theory and Applications, vol. 8, no. 2, pp. 259-278, 2003.

[14] S.-M. Jung and K.-S. Lee, "Hyers-Ulam stability of first order linear partial differential equations with constant coefficients," Mathematical Inequalities \& Applications, vol. 10, no. 2, pp. 261266, 2007.

[15] B. Hegyi and S.-M. Jung, "On the stability of heat equation," Abstract and Applied Analysis, vol. 2013, Article ID 202373, 4 pages, 2013.

[16] S.-M. Jung, "On the stability of the heat equation with an initial condition," Journal of Inequalities and Applications, vol. 2013, no. 475, 6 pages, 2013.

[17] G. Wang, M. Zhou, and L. Sun, "Hyers-Ulam stability of linear differential equations of first order," Applied Mathematics Letters, vol. 21, no. 10, pp. 1024-1028, 2008.

[18] A. D. Polyanin and V. F. Zaitsev, Handbook of Exact Solutions for Ordinary Differential Equations, Chapman \& Hall/CRC, 2nd edition, 2003.

[19] S.-M. Jung, "Hyers-Ulam stability of linear differential equations of first order, II," Applied Mathematics Letters, vol. 19, no. 9, pp. 854-858, 2006.

[20] D. Popa and I. Raşa, "On the Hyers-Ulam stability of the linear differential equation," Journal of Mathematical Analysis and Applications, vol. 381, no. 2, pp. 530-537, 2011.

[21] Soon-Mo Jung and Seungwook Min, "Stability of the Wave Equation with a Source," Journal of Function Spaces, vol. 2018, Article ID 8274159, 4 pages, 2018. 


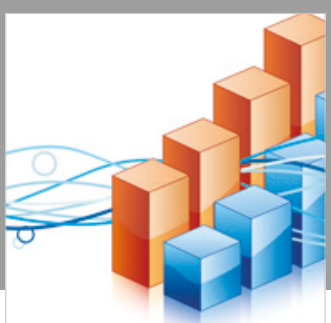

Advances in

Operations Research

\section{-n-m}
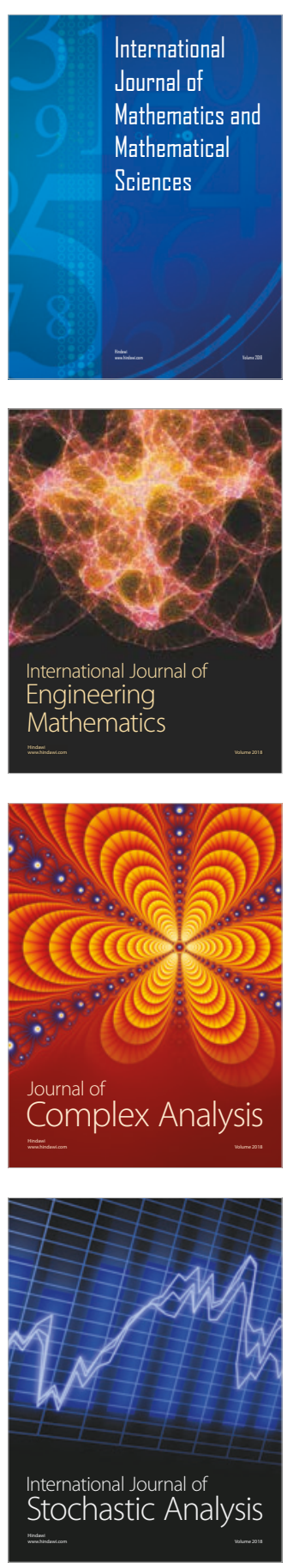
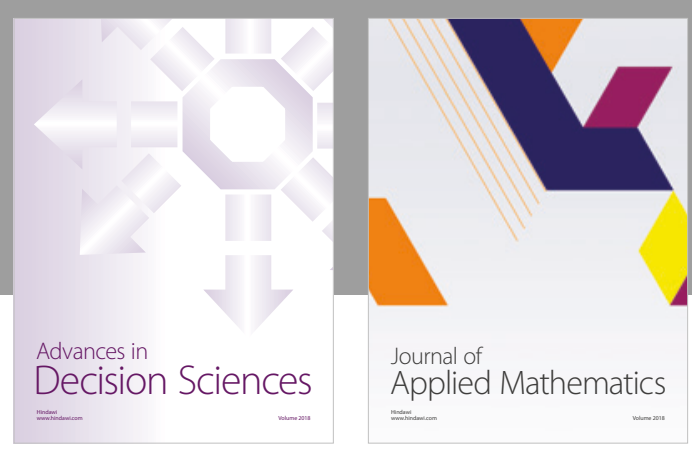

Journal of

Applied Mathematics
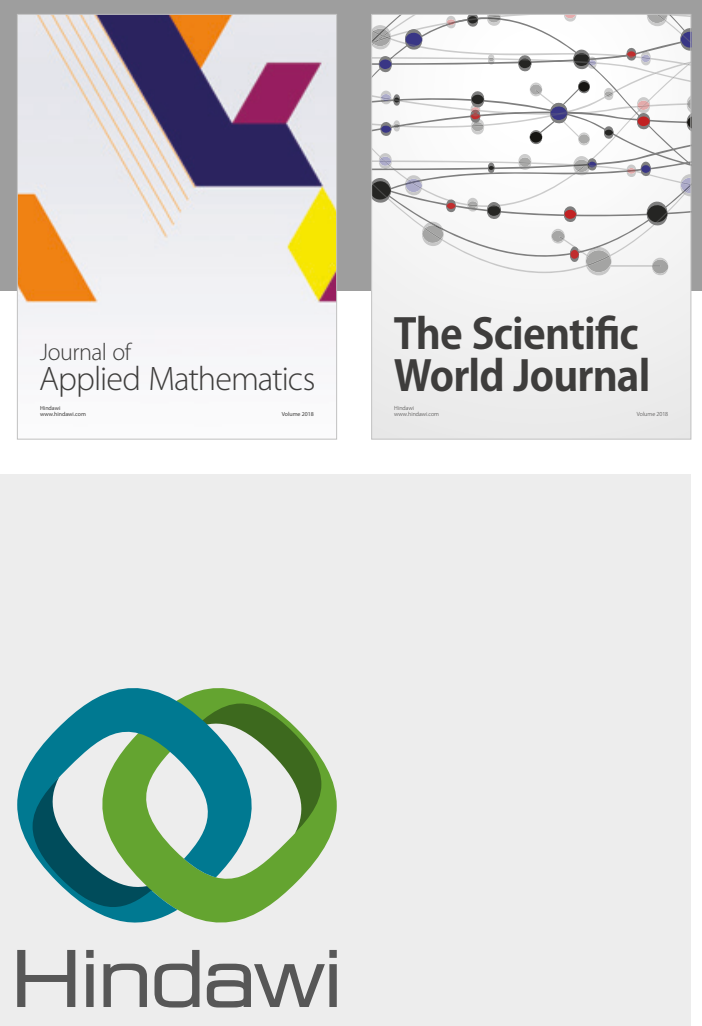

Submit your manuscripts at

www.hindawi.com

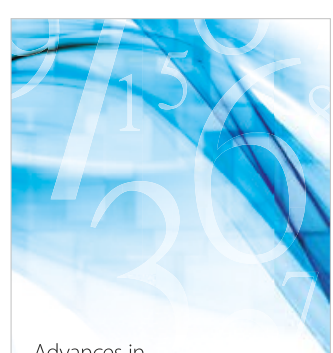

Advances in
Numerical Analysis
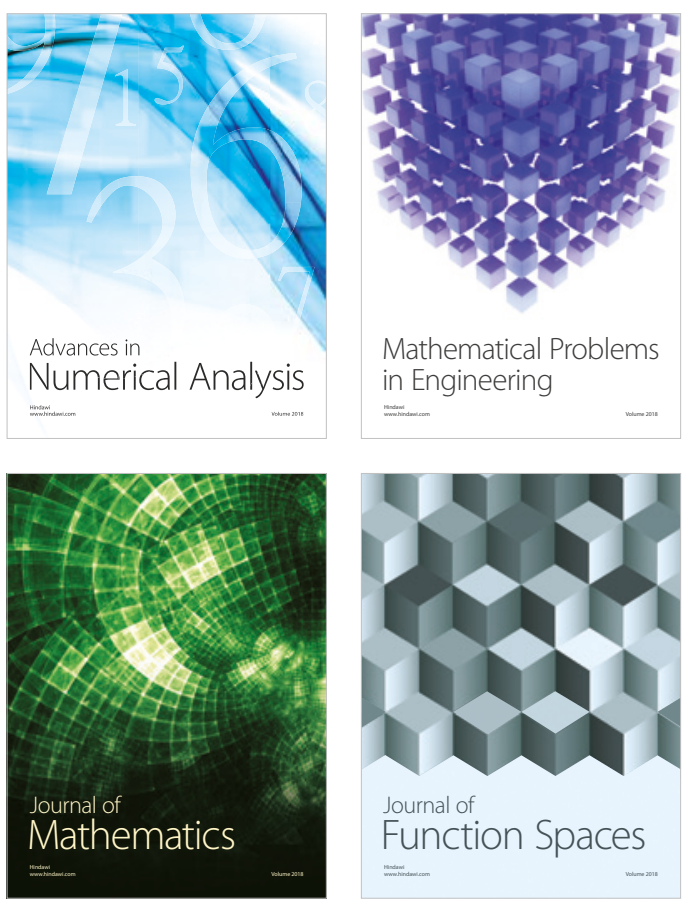

Mathematical Problems in Engineering

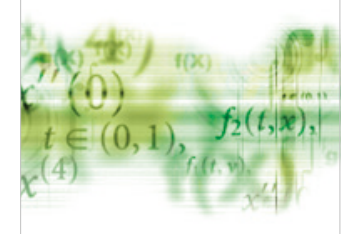

International Journal of

Differential Equations

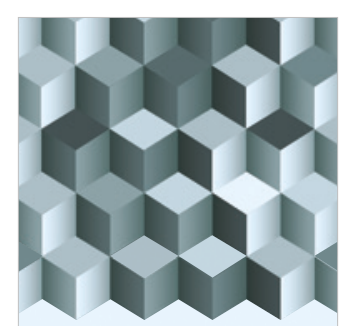

Journal of

Function Spaces

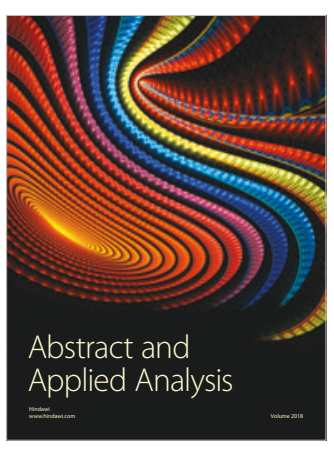

The Scientific

World Journal

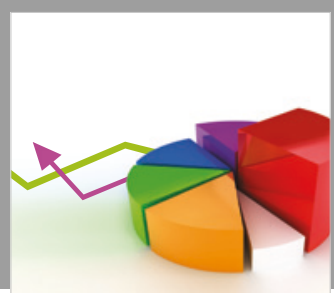

Journal of

Probability and Statistics
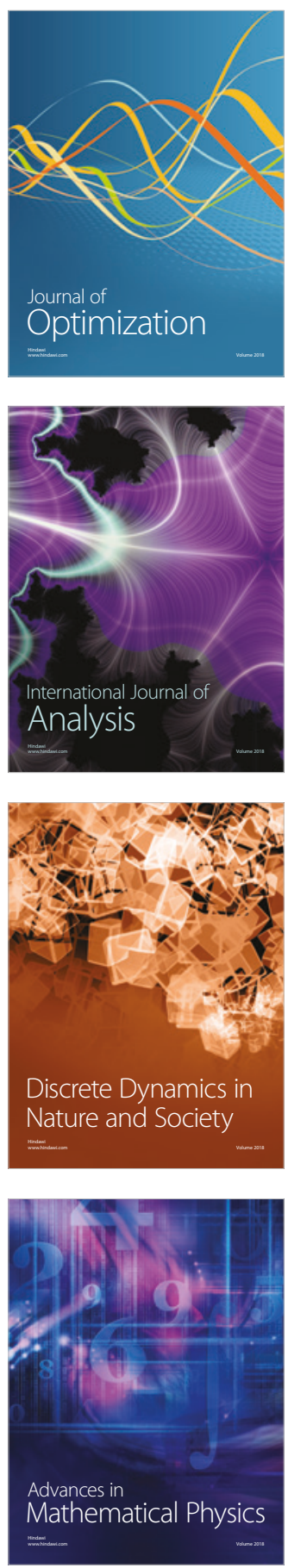